\title{
SPEED LIMIT ON NEPTUNE MIGRATION IMPOSED BY SATURN TILTING
}

\author{
GwenaëL Boué, Jacques Laskar and Petr Kuchynka \\ Astronomie et Systèmes Dynamiques, IMCCE-CNRS UMR8028, Observatoire de Paris, UPMC, 77 Av. \\ Denfert-Rochereau, 75014 Paris, France \\ boue@imcce.fr
}

October 30,2018

\begin{abstract}
In this Letter, we give new constraints on planet migration. They were obtained under the assumption that Saturn's current obliquity is due to a capture in resonance with Neptune's ascending node. If planet migration is too fast, then Saturn crosses the resonance without being captured and it keeps a small obliquity. This scenario thus gives a lower limit on the migration time scale $\tau$. We found that this boundary depends strongly on Neptune's initial inclination. For two different migration types, we found that $\tau$ should be at least greater than $7 \mathrm{Myr}$. This limit increases rapidly as Neptune's initial inclination decreases from 10 to 1 degree. We also give an algorithm to know if Saturn can be tilted for any migration law.
\end{abstract}

Subject headings: celestial mechanics

\section{Introduction}

It is now well accepted that the Solar System was more compact after the protoplanetary gas disk dissipated. Then planets migrated due to interactions with the primordial planetesimal belt. The Nice model $>$ Gomes et al. 2005; Tsiganis et al. 2005; Morbidelli et al. (2005) gives a unified scenario of this planetary migration, but it is still not fully constrained. For example, the Nice model allowed two possible classes of late evolution (Nesvorný et al. 2007). In the first one, called "class MA", Neptune is scattered to 22-25 AU and reaches its final orbit by slowly migrating over more than 5 AU. In the second class, labeled "DE", Neptune is placed to its current orbital distance with large eccentricity $\approx 0.3$ and then slowly circularizes. Besides, the Nice model does not constrain inclinations, and the time scale of this late evoTution is uncertain. Nevertheless, constraints on the miFration time scale were obtained from the distribution of the Kuiper belt on the one hand (Murrav-Clay \& Chiang 2005), and from the distribution of the main asteroid belt on the other (Minton \& Malhotra 2009). They both assumed an MA migration type without long-term evolution of eccentricities and inclinations. The former obtained a migration time scale $\tau$ between 1 and $10 \mathrm{Myr}$, and the latter found $\tau \lesssim 0.5$ Myr.

In this Letter, we aim at giving new constraints based on Saturn tilting (Ward \& Hamilton 2004; Hamilton \& Ward 2004). According to Ward and Hamilton, Saturn's large obliquity, $\epsilon=26.73919 \mathrm{deg}$ (Helled et al. 2009), is due to a resonance capture between its spin axis and Neptune's orbit. Given the large uncertainties on Saturn's precession rate $-0.75 \pm 0.21^{\prime \prime} \cdot \mathrm{yr}^{-1}$ (Ward \& Hamilton 2004), the more accurate regression of Neptune's orbit plane $s_{8}=-0.692^{\prime \prime} \cdot \mathrm{yr}^{-1}$ (Laskar et al. 2004) is indeed included in the errorbars. Ward \& Hamilton (2004) assume that today the two frequencies are equal. In their scenario, the norm of the frequency of Neptune's ascending node was initially larger, and then it captured Saturn's spin axis as it decreased due to Neptune's migration and/or the dissipation of the planetesimal disk (Ward \& Canup 2006). In their numerical model, they took a quasiperiodic model of the Solar System and forced an exponential evolution of the frequency $s_{8}$. Here, we show that Saturn can tilt in both migration classes, and that it gives a lower limit on the migration time scale. This limit depends on Neptune's initial inclination.

A recent paper by Helled et al. (2009) seems to contradict Ward and Hamilton's scenario. It gives a new estimate of Saturn's precession rate $-0.7542 \pm 0.0002^{\prime \prime} \cdot \mathrm{yr}^{-1}$ that is incompatible with a resonance with $s_{8}$. We show that with this value, Saturn can still evolve to its current state but that it is very unlikely. We discuss this result in our conclusion.

\section{Spin axis evolution}

Here we recall the equations of motion of a planet axis and give the current dynamical state of Saturn's spin axis. The evolution of the spin axis $\mathbf{w}$ of a planet in a fixed reference frame $(\mathbf{i}, \mathbf{j}, \mathbf{k})$ where $\mathbf{k}$ is the direction of the total orbital angular momentum, is given by

$$
\frac{d \mathbf{w}}{d t}=-\alpha(\mathbf{n} \cdot \mathbf{w}) \mathbf{n} \times \mathbf{w}
$$

where $\mathbf{n}={ }^{t}\left(n_{x}, n_{y}, n_{z}\right)$ is the normal to the orbit, and $\alpha$ is the precession constant. Without planetary perturbations, $\mathbf{n}$ is fixed and the spin axis $\mathbf{w}$ precesses uniformly around $\mathbf{n}$ with constant obliquity $\cos \epsilon=\mathbf{n} \cdot \mathbf{w}$. However, in a multi-planetary system, $\mathbf{n}$ evolves due to secular interactions. The long-term evolution of $\mathbf{n}$ can be 
approximated by a quasiperiodic expression

$$
n_{x}+i n_{y}=\sum_{k} I_{k} e^{i\left(\nu_{k} t+\varphi_{k}\right)}
$$

where the $\nu_{k}$ (sorted with increasing amplitudes $I_{k}$ ) are combinations of the fundamental frequencies $g_{j}, s_{j}$ (Laskar 1990). For Saturn, $\nu_{2}=s_{8}=-0.692^{\prime \prime} \cdot \mathrm{yr}^{-1}$ and $I_{2}=0.064 \mathrm{deg}$. As the other terms have only very weak effects on the behavior of Saturn's spin axis (Hamilton \& Ward 2004), one can retain this single term in the orbital precession, which makes the problem integrable (Colombo 1966; Henrard \& Murigande 1987). The associated autonomous Hamiltonian, written in a moving reference frame related to the orbital plane, reads

$$
H=-\frac{\alpha}{2}(\mathbf{n} \cdot \mathbf{w})^{2}-\nu(\mathbf{k} \cdot \mathbf{w})
$$

with now $\mathbf{k}={ }^{t}\left(I, 0, \sqrt{1-I^{2}}\right)$ and $\mathbf{n}={ }^{t}(0,0,1)$. The equation of motion, obtained from $d \mathbf{w} / d t=\nabla_{\mathbf{w}} H \times \mathbf{w}$, is

$$
\frac{d \mathbf{w}}{d t}=-\alpha(\mathbf{n} \cdot \mathbf{w}) \mathbf{n} \times \mathbf{w}-\nu \mathbf{k} \times \mathbf{w} .
$$

This system possesses 4 relative equilibriums named Cassini states for which the three axes $\mathbf{w}, \mathbf{n}$ and $\mathbf{k}$ are collinear, and a separatrix delineating 3 zones in the phase space (see Fig. (1). Hereafter, we label the 3 zones after the Cassini state they contain. Saturn's spin axis coordinates in the orbital frame are

$$
\mathbf{w}=\left(\begin{array}{c}
\sin \epsilon \cos \psi \\
\sin \epsilon \sin \psi \\
\cos \epsilon
\end{array}\right)
$$

with $\epsilon=26.73919 \mathrm{deg}$ (Helled et al. 2009) and $\psi=$ $-31 \mathrm{deg}$ (Hamilton \& Ward 2004). As $\psi \neq 0$, the system is not in a Cassini state. Given the coordinates of $\mathbf{w}$, if $-\alpha \cos \epsilon \in[-0.730,-0.666]^{\prime \prime} \cdot \mathrm{yr}^{-1}$ Ward \& Hamilton 2004) then Saturn's spin axis is in resonance around Cassini state 2 with a libration amplitude larger than $31 \mathrm{deg}$ (see Fig 1), else it is in circulation around either Cassini state 1 (see Fig 1a) or Cassini state 3. Literature gives three different values of Saturn's precession rate. Two of them, $-0.74 \pm 0.7^{\prime \prime} \cdot \mathrm{yr}^{-1}$ (French et al. 1993) and $-0.75 \pm 0.21^{\prime \prime} \cdot \mathrm{yr}^{-1}$ (Ward \& Hamilton 2004) are compatible with a libration in zone 2, whereas the third one, $-0.7542 \pm 0.0002^{\prime \prime} \cdot \mathrm{yr}^{-1}$ (Helled et al. 2009), constrains Saturn's axis to circulate in zone 1. In the following, we study these two cases. In Case I, we use the precession constant given by Helled et al. (2009), and in Case II we set $\alpha$ such that $-\alpha \cos \epsilon=s_{8}=-0.692^{\prime \prime} \cdot \mathrm{yr}^{-1}$. In our numerical integrations detailed below, we take into account the dependences of $\alpha$ in Saturn's semi-major axis and eccentricity.

\section{Orbital evolution}

We integrate the secular equations of motion derived from the Hamiltonian of Laskar \& Robutel (1995) written up to degree 4 in inclinations and eccentricities. In order to fit to the present value of $s_{8}$ (Laskar et al. 2004), a small constant offset $\delta s_{8}=-0.00342^{\prime \prime} \cdot \mathrm{yr}^{-1}$ is added in the model. This offset was obtained by frequency analysis
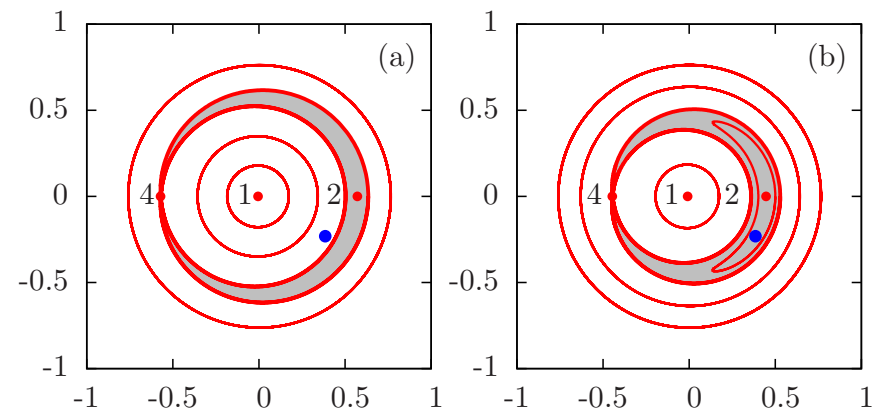

Fig. 1.- Projection of the spin axis $\mathbf{w}$ (5) in the orbital frame (in abscissae $\sin \epsilon \cos \psi$, in ordinate $\sin \epsilon \sin \psi$ ). (a) Case I with $\alpha=0.845^{\prime \prime} \cdot \mathrm{yr}^{-1}$ (Helled et al. 2009). (b) Case II with $\alpha=0.775^{\prime \prime} \cdot \mathrm{yr}^{-1}$ (Ward \& Hamilton 2004). Cassini state 3 corresponds to a retrograde rotation of Saturn and is not represented in these figures. The current position of Saturn's spin axis is represented by a large filled circle. The small filled circles are Cassini states and the curves are energy contours. The bold curve is the separatrix that delineates the libration area in grey.

(Laskar 1990) of our analytical model (Table 1). For the class "MA", we consider only the last 3 AU migration of Neptune. When Neptune was closer to the Sun, the frequency $s_{8}$ was too large to have any effect on Saturn's axis. Migration is simulated by an additional force leading to the following exponential law,

$$
a(t)=a_{0}+\Delta a\left(1-e^{-t / \tau}\right),
$$

with $\Delta a=+0.1,-0.3,-1.3,-3$ AU respectively for Jupiter, Saturn, Uranus, and Neptune. It is scaled from Minton \& Malhotra (2009) and it is in agreement with the full integration of Tsiganis et al. (2005). In the same way, for the class "DE" we apply an external force that gives a long-term exponential evolution of Neptune eccentricity starting at $e_{0}=0.3$ and finishing at its current value. For both classes, we did integrations with constant Neptune inclination, and others with an exponential damping with the same $\tau$. For each value of $\tau$, an integration in the past is done to obtain initial conditions for the orbital coordinates. Saturn's initial obliquity is then set to $\epsilon_{0}=1.5 \mathrm{deg}$.

Table 1: Secular Frequencies Associated with the Precession of the Ascending Nodes.

\begin{tabular}{lrr}
\hline Frequency & Laskar et al. $(2004)$ & Secular integration \\
\hline$s_{5}$ & -0.000 & -0.000 \\
$s_{6}$ & -26.348 & -26.569 \\
$s_{7}$ & -2.993 & -2.996 \\
$s_{8}$ & -0.692 & -0.689 \\
\hline
\end{tabular}

We now look at the effect of the dissipation of the remaining primordial planetesimal belt. Following a suggestion of Morbidelli (2009), the mass $m_{K}$ of the planetesimal belt in the class "MA" is estimated by energy conservation as follows. Initially, planetesimals are distributed following Morbidelli et al. (2004, fig. 1) and during Neptune 


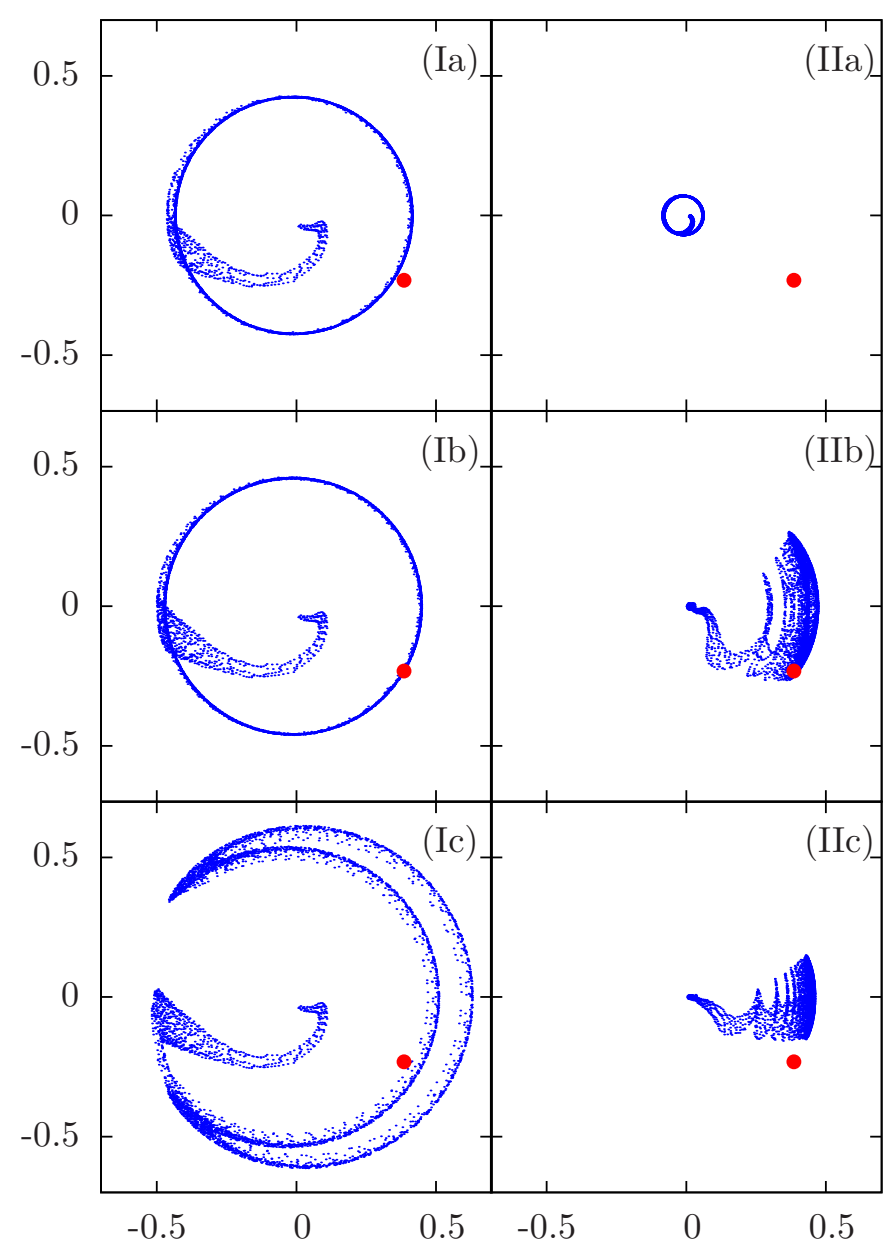

Fig. 2.- Results for the MA type migration. Projection of Saturn's spin axis on the invariant plane in a frame rotating at Neptune regression frequency (in abscisse $\sin \theta \cos (\phi-\Omega)$, in ordinate $\sin \theta \sin (\phi-\Omega)$ ) (see Eq. 9). The filled circle represents its current position. Subfig. Ia, Ib and Ic, Case I with $\tau=180 \mathrm{Myr}$ and $\psi=108.674,108.675469,108.675475 \mathrm{deg}$. Subfig. IIa, IIb and IIc, Case II with $\tau=20,200,300$ Myr and $\psi=0$ deg.

migration, planetesimals move from their initial position to Uranus' orbit. This leads to $m_{K}=1.7 \pm 0.1 M_{\oplus}$. During planet migration with a planetesimal disk, we force an exponential decrease of the planetesimal belt mass with the same time scale as the semi-major axis one. To first order, the averaged effect of a planetesimal of mass $m_{i}$ and semi-major axis $a_{i}$ on Neptune's nodal precession rate is

$$
\delta s_{8}\left(m_{i}, a_{i}\right)=-\frac{n_{N}}{4}\left(\frac{m_{i}}{m_{0}}\right)\left(\frac{a_{N}}{a_{i}}\right)^{2} b_{3 / 2}^{(1)}\left(a_{N} / a_{i}\right)
$$

with $n_{N}$ and $a_{N}$ being respectively Neptune mean motion and semi-major axis, and $m_{0}$ the mass of the Sun. We model the planetesimal belt by a single annulus with semimajor axis $a_{K}$ such that

$$
\delta s_{8}\left(m_{k}, a_{k}\right)=\sum_{i} \delta s_{8}\left(m_{i}, a_{i}\right)
$$

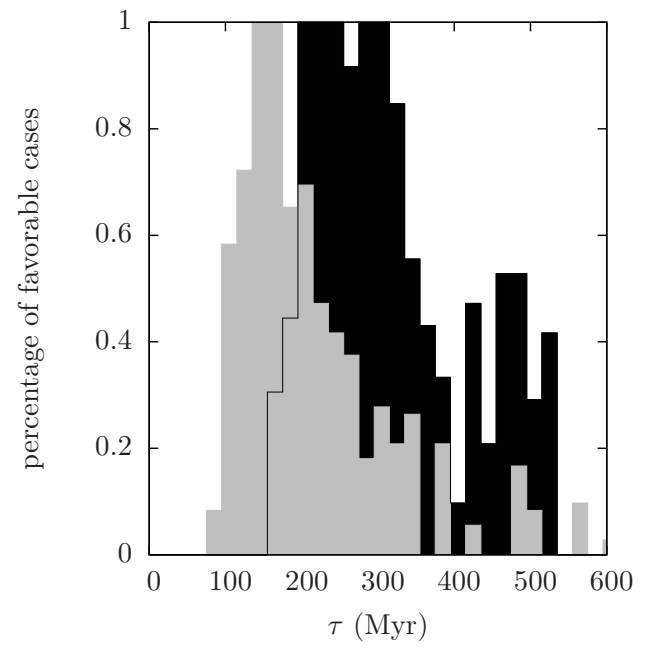

Fig. 3.- Probability that Saturn librates in zone 2 with an amplitude larger than $31 \mathrm{deg}$ as a function of the migration time scale $\tau$ in Case II. MA migration type in grey and DE migration type in black.

Using the mass distribution of Morbidelli et al. (2004), we found $a_{k}=60 \pm 5 \mathrm{AU}$. We run numerical integrations with and without a 2 Earth mass primordial planetesimal belt and found that the constraints on the migration time scales were unchanged. In the following, we give only the results of our integrations without a planetesimal disk.

\section{Results}

In Case I, Saturn's spin axis circulates around Cassini state 1 with a large obliquity (Fig. 11). To show whether it is compatible with the Ward \& Hamilton (2004) scenario or not, we did several numerical integrations without long-term evolution of Neptune inclination and enumerated those ending in zone 1 with an obliquity larger than or equal to $26.73919 \mathrm{deg}$. We first considered the class MA of the Nice model and varied the migration time scale $\tau$ from $100 \mathrm{Myr}$ to $600 \mathrm{Myr}$ every $10 \mathrm{Myr}$. Then, for each value of $\tau$, we searched the range of the initial precession angle $\psi$ for which the final state corresponds to our criterion. We found only 3 values for $\tau$ satisfying the criterion: $\tau \in\{170,180,190\}$ Myr. In each case, the range of possible values for $\psi$ is extremely small $\Delta_{\psi} \lesssim 10^{-5} \mathrm{deg}$ (Fig. 2] Iabc). Thus assuming an equiprobable initial phase, the probability to find Saturn in its current state through this mechanism is less than $3 \times 10^{-8}$ for any of the three selected $\tau$. With a DE migration type, the widths of the initial longitude intervals $\Delta_{\psi}$ are identical. The only changes are in the values of the migration time scale $\tau$ leading to the large obliquity circulation state: $\tau \in\{150,260,290,310,320\}$ Myr. We discuss the implications of these results in the conclusion.

In Case II, Saturn spin axis is presently in resonance with Neptune's ascending node. In that case, planet migration must be slow enough for the capture to occur, but if it is too slow, then the evolution becomes adiabatic and the libration amplitude is too small (less than $31 \mathrm{deg}$ ) (Fig 2, IIc). This latter constraint disap- 


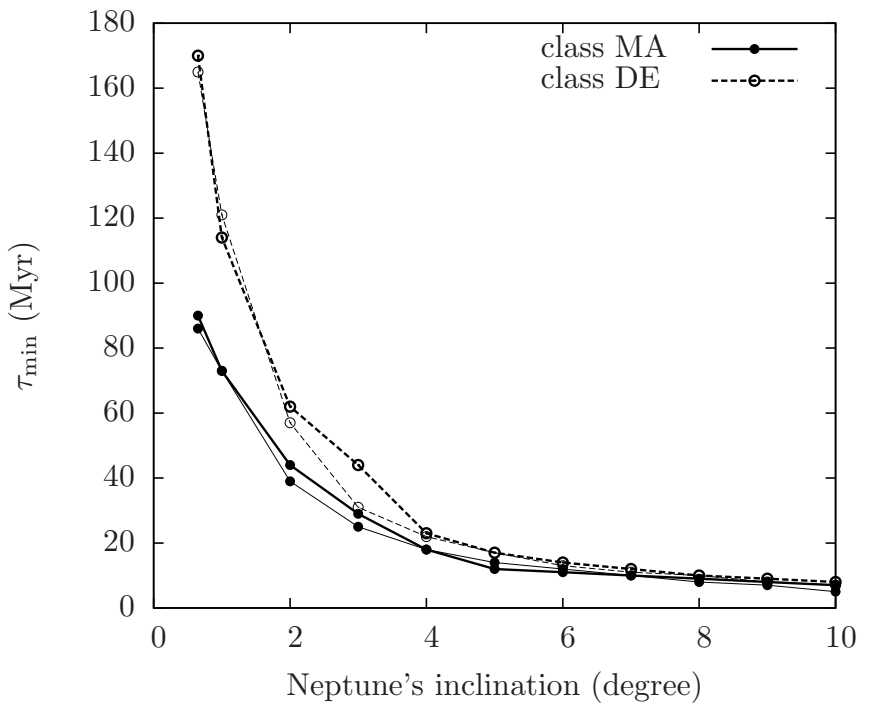

Fig. 4.- Minimal migration time scale as a function of Neptune's initial inclination for both migration types: MA (solid line) and DE (dashed line). Bold curves are results of numerical integrations. Thin curves were obtained by the algorithm described at the end of Section 5 .

pears if the precapture obliquity is larger than $4.5 \mathrm{deg}$ (Ward \& Hamilton 2004). We performed 2100 integrations for each of the two migration types MA and DE, $\tau$ going from 10 to $600 \mathrm{Myr}$ every $10 \mathrm{Myr}$ and $\psi$ between 0 and $350 \mathrm{deg}$ every $10 \mathrm{deg}$. The results are summarized in Fig. 3. MA type in grey and DE type in black. Probabilities are now significant and reach 1 for a few time scales. We see clear lower limits, $\tau \geq 90$ Myr (resp. $\tau \geq 170 \mathrm{Myr}$ ) for the MA (resp. DE) migration type. The difference in the results between the two Nice model classes comes mainly from the different dependence of the semi-major axis and the eccentricity on Neptune's regression frequency. In all these integrations, Neptune's inclination does not undergo long-term evolution. However, the amplitude $I_{2}$ of Saturn orbital quasiperiodic motion (2) is proportional to Neptune inclination. In Section 5. we show that the higher the inclination amplitude is, the faster a planet can be tilted. We thus studied the minimum time scale, for which Saturn's axis ends in zone 2 with a libration amplitude larger than $31 \mathrm{deg}$, as a function of Neptune's initial inclination (Fig. 4, bold curves). In both migration classes, $\tau_{\min }$ decreases rapidly to $\approx 20$ Myr when Neptune's initial inclination increases to $4 \mathrm{deg}$ and then it decreases slowly down to $\approx 7$ Myr when Neptune's inclination goes to $10 \mathrm{deg}$.

\section{Fastest tilting}

In this section, we compute analytically the minimal time required to tilt a planet as a function of its inclination $I(t)$. We give also an algorithm to check whether Saturn can be tilted or not for a given migration.

We call $\theta$ the inclination of a planet equator relative to the invariant plane. As Saturn current inclination is small relative to its obliquity $\epsilon$, the two angles $\theta$ and $\epsilon$ are similar. Let $\Phi$ and $\Omega$ be the longitude of the ascending node of the equator and of the orbit in the invariant plane. We have

$$
\mathbf{w}=\left(\begin{array}{c}
\sin \theta \sin \Phi \\
-\sin \theta \cos \Phi \\
\cos \theta
\end{array}\right)_{(\mathbf{i}, \mathbf{j}, \mathbf{k})} \mathbf{n}=\left(\begin{array}{c}
\sin I \sin \Omega \\
-\sin I \cos \Omega \\
\cos I
\end{array}\right)_{(\mathbf{i}, \mathbf{j}, \mathbf{k})}
$$

and

$$
\begin{aligned}
\mathbf{n} \cdot \mathbf{w} & =\sin \theta \sin I \cos (\Phi-\Omega)+\cos \theta \cos I, \\
(\mathbf{n} \times \mathbf{w}) \cdot \mathbf{k} & =-\sin \theta \sin I \sin (\Phi-\Omega) .
\end{aligned}
$$

Thus, from (1),

$\frac{d \cos \theta}{d t}=\alpha \sin \theta \cos \theta \sin I \cos I[1+\gamma \cos (\Phi-\Omega)] \sin (\Phi-\Omega)$

where $\gamma=\tan \theta \tan I$ can vary from 0 to infinity depending on the value of the obliquity. We now choose $\Omega$ that maximizes this time derivative as a function of $I$ and $\theta$. Doing so, we ensure that it is not possible to have a faster evolution of the equator inclination $\theta$. This leads to

$$
\cos (\Phi-\Omega)=\frac{-1+\sqrt{1+8 \gamma^{2}}}{4 \gamma} .
$$

Substituting this expression in (11) gives the maximal speed $\Theta_{\max }$ such that $d \theta / d t \leq \Theta_{\max }$

$$
\Theta_{\max }=\frac{\alpha \sqrt{2}}{16}|\cos \theta \sin 2 I| \frac{\left(\sqrt{1+8 \gamma^{2}}+3\right)^{3 / 2}}{\left(\sqrt{1+8 \gamma^{2}}+1\right)^{1 / 2}} .
$$

After some calculus, it can be shown that $\Theta_{\max }$ is an increasing function of $\tan I$. Thus, if the only constraint on the orbit inclination amplitude is an upper limit $I_{\max }<$ $\pi / 2$, the fastest evolution is obtained for $I=I_{\max }$. In two asymptotic cases, the expressions of $\Theta_{\max }$ are simpler. For $I \ll|\pi / 2-\theta|$ or $\theta \ll|\pi / 2-I|$, we have $|\gamma| \ll 1$ and thus

$$
\Theta_{\max } \approx \frac{\alpha}{2}|\cos \theta \sin 2 I| \text {. }
$$

In the other case, if $|\theta-\pi / 2| \ll I$ or $|I-\pi / 2| \ll \theta$, the parameter $\gamma$ is arbitrarily large and (13) becomes

$$
\Theta_{\max } \approx \frac{\alpha}{2} \sin \theta \sin ^{2} I
$$

Using the approximation for small angles $|\gamma| \ll 1$ (14), the minimum time $t_{\min }$ required to bring $\theta$ from 0 to $\theta_{\text {end }}$ at constant inclination amplitude $I$ is

$$
t_{\min }=-\frac{2}{\alpha \sin 2 I} \ln \left|\tan \left(\frac{\pi}{4}-\frac{\theta_{\text {end }}}{2}\right)\right| .
$$

In Saturn's case, the amplitude of the mode responsible for the tilt is $I_{2}$. Whenever Neptune's inclination is less than $10 \mathrm{deg}, I_{2}$ remains below $0.9 \mathrm{deg}, \gamma \leq 8 \times 10^{-3}$ and Expressions (14, 16) are valid. This minimum time $t_{\min }$ decreases with the inclination amplitude $I$. For example, with $I=I_{2}=0.064 \mathrm{deg}$, Equation (16) gives $t_{\min }=105$ Myr in Case I and $t_{\min }=115$ Myr in Case 2. 
From this study, it is possible to check whether Saturn's axis can be tilted or not for a given migration. Let $\theta_{2}$ be the value of $\theta$ at the Cassini state 2. $\theta_{2}$ and its time derivative $\Theta_{2}=\dot{\theta}_{2}$ are functions of orbital parameters through $I(t), \nu(t)=s_{8}(t)$ and $\alpha(t)$. During a tilt, $\theta$ oscillates around the increasing $\theta_{2}$ and $\Theta=\dot{\theta}$ reads

$$
\Theta=\Theta_{2}+A \omega_{\text {lib }} \sin \left(\omega_{\text {lib }} t+\varphi\right)
$$

where $A$ and $\varphi$ are respectively the libration amplitude and a phase, and $\omega_{\text {lib }}$ is the libration amplitude given by Hamilton \& Ward (2004)

$$
\omega_{\text {lib }}=\sqrt{-\alpha \nu \sin \theta \sin I}
$$

For a given migration, Saturn's axis can tilt if and only if there exist $A$ and $\phi$ such that $\Theta \leq \Theta_{\max }$ (14)and 17) during all the evolution. Replacing $\theta$ by $\theta_{2}$ in (14) and 18), one obtains a criterion that depends only on orbital parameters. We applied this criterion on the systems studied in Section 4. For each value of Neptune's initial inclination, we integrated once the system with a given $\tau$. Then, we rescaled the derivatives $\Theta$ for different value of $\tau$ until the criterion is verified. The resulting values of $\tau_{\min }$ are displayed in Fig. (4, thin curves).

\section{Conclusions}

First of all, we see that the Helled et al. (2009) precession constant is incompatible with the Ward \& Hamilton (2004) scenario. This is a robust result. Helled et al. (2009) obtained Saturn's precession constant from an empirical model of its internal structure. They used Saturn mass, radius and gravitational coefficients $J_{2}, J_{4}$, and $J_{6}$ to fit a density profile represented by a sixth degree polynomial. From this density profile they derived the normalized axial moment of inertia $\gamma$ directly related to the precession constant. Our results suggest, rather, considering $\gamma$ as an additional independent parameter to better constrain Saturn's interior. If Saturn is actually in libration in zone 2 then $0.2257<\gamma<0.2438$ Ward \& Hamilton 2004).

Assuming the Hamilton \& Ward (2004) precession constant, Saturn's spin axis is likely to evolve toward a libration in zone 2 whatever the migration class is as long as the time scale $\tau$ is sufficiently large. We found a strong dependence between the minimum time scale $\tau_{\text {min }}$ and Neptune's inclination. Thus, an external constraint on the speed limit of Neptune migration may also constrain its inclination. For instance, the upper boundary obtained by Murray-Clay \& Chiang (2005) is $\tau \leq 10$ Myr. In that case, our results show that under the hypothesis of Section 3. the initial inclination of Neptune's orbit must have been larger than $7 \mathrm{deg}$. On the other side, in all our studied cases, the minimum time scale must be at least greater than 7 Myr, whereas Minton \& Malhotra (2009) found $\tau \lesssim 0.5$ Myr. This contradiction may be raised if one considers different evolution laws for the semi-major axes, eccentricities, and/or inclinations. In that scope, we have given in Section 5 an algorithm to know if Saturn can be tilted for any migration law.

\section{REFERENCES}

Colombo, G. 1966, AJ, 71, 891
French, R. G., Nicholson, P. D., Cooke, M. L., Elliot, J. L., Matthews, K., Perkovic, O., Tollestrup, E., Harvey, P., Chanover, N. J., Clark, M. A., Dunham, E. W., Forrest, W., Harrington, J., Pipher, J., Brahic, A., Grenier, I., Roques, F., \& Arndt, M. 1993, Icarus, 103, 163

Gomes, R., Levison, H. F., Tsiganis, K., \& Morbidelli, A. 2005, Nature, 435, 466

Hamilton, D. P., \& Ward, W. R. 2004, AJ, 128, 2510

Helled, R., Schubert, G., \& Anderson, J. D. 2009, Icarus, 199, 368

Henrard, J., \& Murigande, C. 1987, Celestial Mechanics, 40, 345

Laskar, J. 1990, Icarus, 88, 266

Laskar, J., \& Robutel, P. 1995, Celestial Mechanics and Dynamical Astronomy, 62, 193

Laskar, J., Robutel, P., Joutel, F., Gastineau, M., Correia, A. C. M., \& Levrard, B. 2004, A\&A, 428, 261

Minton, D. A., \& Malhotra, R. 2009, Nature, 457, 1109

Morbidelli, A. 2009, personal communication

Morbidelli, A., Emel'yanenko, V. V., \& Levison, H. F. 2004, MNRAS, 355, 935

Morbidelli, A., Levison, H. F., Tsiganis, K., \& Gomes, R. 2005, Nature, 435, 462

Murray-Clay, R. A., \& Chiang, E. I. 2005, ApJ, 619, 623

Nesvorný, D., Vokrouhlický, D., \& Morbidelli, A. 2007, AJ, 133, 1962

Tsiganis, K., Gomes, R., Morbidelli, A., \& Levison, H. F. 2005, Nature, 435, 459

Ward, W. R., \& Canup, R. M. 2006, ApJ, 640, L91

Ward, W. R., \& Hamilton, D. P. 2004, AJ, 128, 2501

This 2-column preprint was prepared with the AAS IATEX macros v5.2. 